\title{
Effects of behavioural exercise therapy on the effectiveness of a multidisciplinary rehabilitation for chronic non-specific low back pain: Study protocol for a randomised controlled trial
}

Jana Hofmann, Stefan Peters, Wolfgang Geidl, Christian Hentschke and Klaus Pfeifer*

\begin{abstract}
Background: In Germany, a multidisciplinary rehabilitation named "behavioural medical rehabilitation" (BMR) is available for treatment of chronic low back pain (clbp). A central component of BMR is standard exercise therapy (SET), which is directed mainly to improve physical fitness. There is a need to address psychosocial factors within SET and therefore to improve behavior change with a focus on the development of self-management skills in dealing with clbp. Furthermore, short-term effectiveness of BMR with a SET has been proven, but the impact of a behavioural exercise therapy (BET) for improvement of the long-term effectiveness of BMR is unclear.
\end{abstract}

Methods/design: To compare the effectiveness of two exercise programs with different approaches within BMR on the effects of BMR a prospective randomized controlled trial $(R C T)$ in two rehabilitation centres will be performed. 214 patients aged 18-65 with clbp will be, based on an "urn randomisation"-algorithm, randomly assigned to a BMR with SET (function-oriented, $n=107$ ) and BMR with BET (behaviour-oriented, $n=107$ ). Both exercise programs have a mean duration of 26 hours in three weeks and are delivered by a limited number of not-blinded study therapists in closed groups with six to twelve patients who will be masked regarding study group. The main differences of BET lie in its detailed manualised program with a theory-based, goal-orientated combination of exercise, education and behavioural elements, active participation of patients and consideration of their individual preferences and previous experiences with exercise. The primary outcome is functional ability assessed with the Hannover Functional Ability Questionnaire directly before and after the rehabilitation program, as well as a six and twelve-month follow-up.

Discussion: This RCT is designed to explore the effects of BET on the effectiveness of a BMR compared to a BMR with SET in the management of patients with clbp. Methodological challenges arise from conducting a RCT within routine health care as well as from ensuring high treatment integrity. Findings of this study might contribute to a better understanding of the mechanism of action of BMR and the special effects of BET and may be used to improve the quality of these interventions in routine care, therefore reducing the burden to patients with disabling clbp.

Trial registration: Current controlled trials NCT01666639

Keywords: Chronic non-specific low back pain, Exercise therapy, Behavioural exercise therapy, Multidisciplinary rehabilitation, Self-management, Physical activity

\footnotetext{
* Correspondence: klaus.pfeifer@sport.uni-erlangen.de

Institute of Sport Science and Sport, Friedrich-Alexander-University Erlangen-

Nürnberg, Gebbertstr. 123b, D-91058 Erlangen, Germany
} 


\section{Background}

Non-specific low back pain is one of the leading health problems regarding its medical and economic impact worldwide [1-4]. This is also the case in Germany with high substantial economic burden of an estimated $€ 48.96$ billion total costs related to low back pain for the entire German adult population aged 18 to $75[5,6]$.

While the lifetime prevalence of low back pain is reported to be up to $84 \%$, little scientific evidence is available for the prevalence of disabling chronic low back pain $[4,7]$. In a multiregional survey in the adult population in Germany reported more than $11 \%$ disabling chronic low back pain [5]. Clbp is also one of the most frequent conditions in traditional multidisciplinary inpatient rehabilitation in Germany [8].

Intensive multidisciplinary rehabilitation is recommended as first-line treatment for the management of disabling clbp $[4,7,9]$. It has been proven to be effective in the short term regarding pain intensity and functional disability compared to no treatment or waiting list controls [10]. But, there is a lack of evidence regarding the long-term effectiveness of such programs. Behavioural medical rehabilitation (BMR) in Germany is an intensive multidisciplinary inpatient rehabilitation program with a theory-based biopsychosocial approach [11]. In contrast to the traditional multidisciplinary inpatient rehabilitation, BMR is explicitly directed at orthopedic patients who show significant psychosocial risk factors for the chronification of musculoskeletal disorders. In a randomised controlled trial, the BMR had a positive short-term effect on depression and sustainable effects on several pain coping competencies [12] compared to traditional inpatient rehabilitation. A meta-analysis of intensified multidisciplinary inpatient rehabilitation for patients with musculoskeletal disorders [13] proved BMR to be superior to usual care regarding a sustainable improvement of the subjective health status. Evidence of long-term effects of BMR on pain and functional disability is lacking. It is also not yet clear which treatment component or combination of treatment components of an intensified rehabilitation regime explains the amount of variance regarding the effects in patients with disabling clbp [14].

A corner-stone is SET, which represents approximately $70 \%$ of all interventions within multidisciplinary rehabilitation, received by orthopedic patients in Germany [15]. SET is mainly based on a functional approach with methods to improve physical fitness [15,16]. Evidence shows that SET compared to usual care improves pain and function in patients with clbp, however the reported effect-sizes are low $[17,18]$.

There is also evidence available confirming that psychosocial factors play a pivotal role in the development of clbp [19-21]. Addressing them specifically within exercise therapy might reduce obstacles to recovery and increased function [22,23]. Especially to improve self-management of back pain and the long-term adherence to regular physical activity [24], a broader theory-based approach with appropriate tools and strategies used by physical therapists within SET seems necessary [25,26]. An integration of biopsychosocial education and behavior change as well as the consideration of patients' individual preferences and the realisation of positive experiences with physical activity seem to be superior to a solely functional approach [17,18,24,26-30].

An enhancement of long-term adherence to physical activity in chronically ill adults via patient-oriented methods directed at motivation and volition (e.g. planning) has been effective [27,31,32]. Conn et al. [27] detected behavior change interventions being effective across medical conditions (effect size 0.45 ) and showed an average increase in the amount of performed physical activity, which was 48 minutes higher than the increase in the control groups. The Review of Jordan et al. [24] examining interventions to improve exercise adherence in musculoskeletal disorders outlined that adherence is not influenced by the kind of exercise being performed (e.g. strength training, endurance-oriented activities). A combination of exercise with cognitive and behavioural strategies seems to be more important to improve adherence to physical activity or exercise and the addition of volitional methods to motivational methods is paramount [24,27,31-33].

There has not been in-depth research about the biopsychosocial effects of theory-based exercise therapy with a behavioural approach, named behavioural exercise therapy (BET), as part of the BMR in the management of disabling clbp. So the impact of a BET for improvement of the effectiveness of BMR is unclear.

\section{The aims of this study}

Therefore the aims of this study are a) the development and implementation of a BET into an existing BMR and b) the evaluation of the effectiveness of BMR with BET compared to BMR with SET in routine health care (short-, mid-, and long-term effects) in the management of patients with disabling clbp.

\section{Methods/design \\ Study design}

To evaluate the effects of BET on the effectiveness of a BMR, a multicentre-study with a prospective randomised controlled design is conducted. This study takes place in two inpatient rehabilitation centres in Germany. Participants with clbp will be randomised into either a) the usual BMR with the SET as the control group (CG) or b) the BMR with the extended BET as the intervention group (IG) 
(see Figure 1). Primary and secondary outcomes will be measured by questionnaires directly before and at the end of the BMR (after approx. four weeks), as well as six and twelve months after completion of the BMR. The following figure shows the study design with appropriate study phases as well as the expected duration (see Figure 2).

\section{Hypotheses}

\section{Primary hypothesis}

In patients with clbp, treatment with BMR+BET will result in a significantly higher increase in functional ability directly at the end, as well as six and twelve months after completion of BMR+BET compared to usual BMR+SET.

\section{Secondary hypothesis}

There are numerous interrelations between selfmanagement of clbp, pain-related cognitions, physical activity, health-related quality of life and the number of back pain episodes [19,21,22,34-37]. For these reasons the following secondary hypotheses are tested:

In patients with clbp, treatment with BMR+BET, compared to usual BMR+SET, will result in a significant:

- Improvement of pain-related cognitions

- Increase of physical activity

- Improvement in health-related quality of life

- Decrease in back pain episodes

- Improvement in pain coping strategies

\section{Study population}

Patients with clbp, who are invited consecutively in the two collaborating rehabilitation centres between January 2012 and January 2013, are eligible by reservation. Chronic non-specific low-back pain is defined as pain persisting for at least three months, localised below the

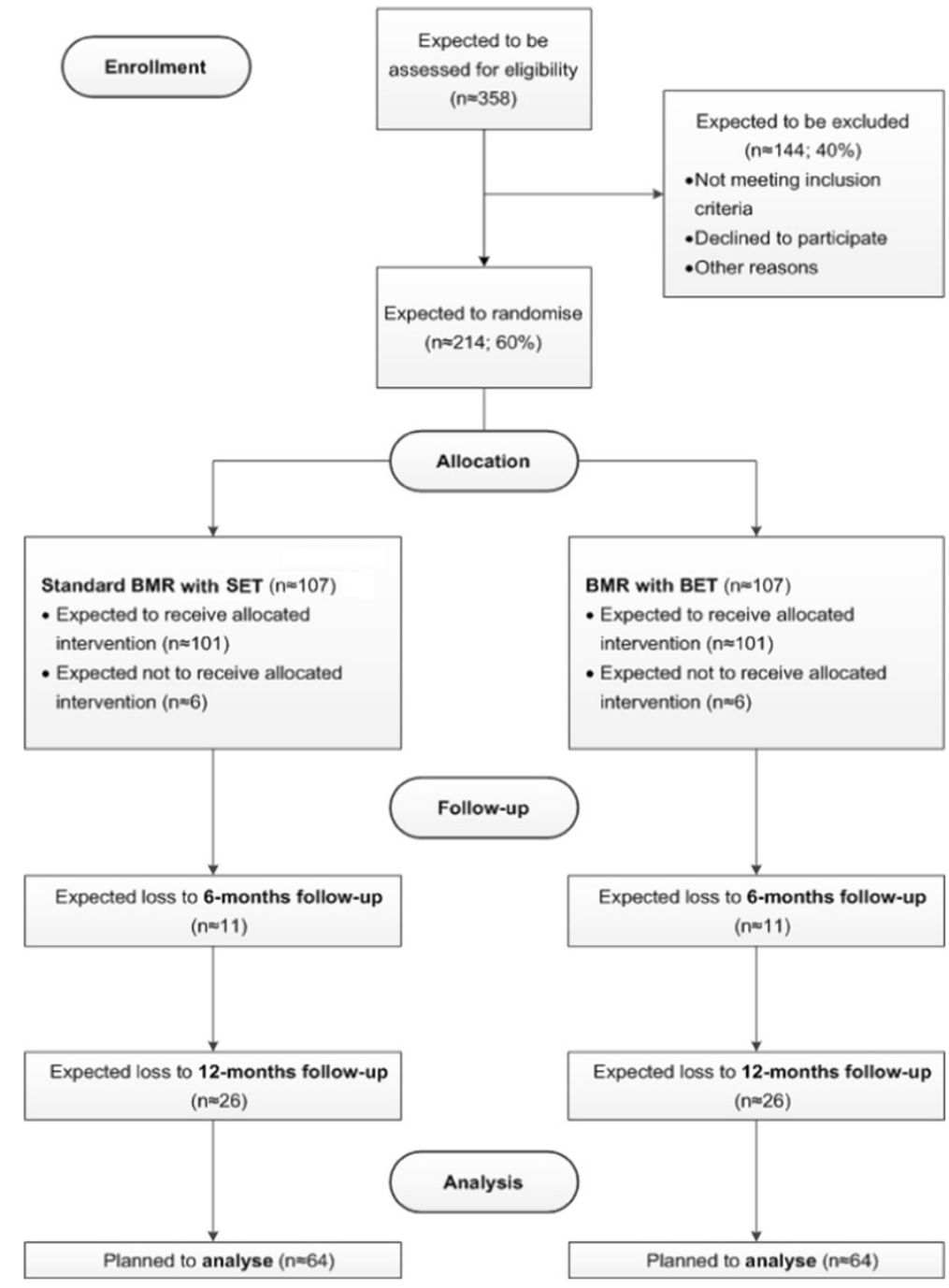

Figure 1 Study Flow Diagram. 
costal margin and above the inferior gluteal folds, without referred leg pain and that is not caused by a known specific pathology [7]. Inclusion criteria are based on the international classification of diseases (ICD-10) in its German modification. Inclusion and exclusion criteria are described in Table 1.

\section{Recruitment procedures}

After submission of the signed consent form of the study participants („informed consent"), patient enrollment takes place in the participating rehabilitation centres based on the assessment of the inclusion and exclusion criteria by the attending physician. Next, patients are randomly assigned to the different treatment groups.

The recruitment procedure is adjusted to each of the centre's internal structures and processes regarding the invitation of patients.

In the rehabilitation centre "Paracelsus-Klinik an der Gande" potential study participants will be identified by a screening of the patient record by the head physician

Table 1 Inclusion and exclusion criteria

\begin{tabular}{|c|c|}
\hline Inclusion & Main diagnosis for a BMR: \\
\hline criteria & -M51.2-M51.9 (other intervertebral disc disorders), \\
\hline \multirow[t]{5}{*}{$(\mathrm{ICD}-10)$} & -M53.8, M53.9 (other specified/unspecified dorsopathies), \\
\hline & $\begin{array}{l}\text {-M54.4-M54.9 (lumbago with sciatica, low back pain, } \\
\text { pain in thoracic spine, other/unspecified dorsalgia), }\end{array}$ \\
\hline & -F45.4 (persistent somatoform pain disorder), \\
\hline & $\begin{array}{l}\text {-F45.41 (chronic pain disorder with somatic and } \\
\text { psychological factors), }\end{array}$ \\
\hline & $\begin{array}{l}\text {-F54 (psychological and behavioural factors associated with } \\
\text { disorders or diseases classified elsewhere) }\end{array}$ \\
\hline \multirow[t]{7}{*}{$\begin{array}{l}\text { Exclusion } \\
\text { criteria }\end{array}$} & $\begin{array}{l}\text { - specific underlying diagnosis of the back pain } \\
\text { (e. g. radicular symptoms, myelopathy) }\end{array}$ \\
\hline & -considerably reduced health status (comorbidities) \\
\hline & -considerable reduction of sight and hearing \\
\hline & -severe psychiatric condition as secondary diagnosis \\
\hline & -age below 18 or over 65 respectively \\
\hline & -lack of ability to speak German \\
\hline & -ongoing application for retirement \\
\hline
\end{tabular}

prior to the invitation to the BMR. Afterwards, eligible patients will obtain written patient information and a consent form about the study and will be asked to participate. After returning the signed consent form within seven days, the patients will be randomised online. The invitation of the randomised patients will take place weekly and in groups according to the group assignment in the respective week of arrival. At the beginning of the rehabilitation program, an information meeting will be held by the head physician for all eligible patients fully explaining the study.

In the rehabilitation centre "Klinik Weser" potential study participants will be identified at the beginning of the BMR in medical admission interviews and will obtain a written consent form. In an information meeting all eligible patients are fully informed about the study primarily by the head physician. On the second day, after returning the signed consent form, the online-based randomisation will take place. Due to spatial and personnelwise requirements, one control and one intervention group will start simultaneously every fourteen days. Therefore, the recruitment of eligible patients will take place in a biweekly rhythm.

In both rehabilitation centres, the group size is limited to twelve participants. If the number of eligible study patients is too small for starting a control or intervention group, the participation of non-eligible patients who do not take part in the study is accepted to reach the necessary group size. At least 40 potentially eligible patients will be treated every month in both rehabilitation centres. Considering a recruitment rate of $60 \%$, 358 eligible patients will have to be asked to participate in the study in order to achieve the required sample size. Based on the assumption that average closed groups consist of six to seven participants, the recruitment period is predefined as a period of twelve months. Both rehabilitation centres confirmed that a sufficient number of eligible patients would exist to achieve the necessary sample size in the study period.

The collection of consent forms, distribution and collection of questionnaires and the delivery of the latter 
to the study team are adjusted to the process of patient admission and discharge in standardised form. The process will be tested and automated in each of the rehabilitation centres during the phase of implementation.

\section{Follow-up phase}

The follow-up questionnaires will be sent to the former participants six and twelve months after the rehabilitation treatment by the university. Postal reminders will be send after three weeks if the questionnaire has not been sent back by patients.

\section{Drop out criteria}

Dropouts are defined as patients who do refuse to participate in the study at the end of the rehabilitation program. These patients will not be contacted after six and twelve months for further evaluation.

Also, dropouts are patients who withdraw the entire study participation and prohibit the use of existing personnel data. In this case, the questionnaire filled out at the beginning of the rehabilitation program must be given to the patient.

If patients don't fill out the questionnaire at the end of the rehabilitation (despite former reminder), they will still receive a questionnaire after six and twelve months, if they don't explicitly refuse or withdrew from the study participation.

\section{Registration of non-responders}

The registration of all eligible patients and the reporting of the number and the sex of eligible patients who do not participate (non-responder analysis) during the time of the study will take place for the identification of selection effects during the recruitment process.

\section{Randomisation}

The randomisation procedure of the participants takes place with an online-based randomisation feature. Therefore, we implement a central data base for electronic data recording which will be used in each of the two rehabilitation centres. An independent administration employee, who is not involved in the study, will use the online-based randomization feature within each rehabilitation centre to register eligible participants via a web application. After registration, the online-based randomisation feature automatically accomplishes the allocation to one of the treatment groups. This system has been developed in a prior study [38] and incorporates the existing national data protection regulations. Clear advantages of this procedure are a prompt randomisation and a concealment of the sequence of allocation. For sequence generation we use an "urn randomisation"-algorithm [39]. It has good statistical properties [40] and can be used to stratify by different criteria. In this study we stratify for "rehabilitation centre" with the two rehabilitation centres and "gender" with two categories. Additionally, a structural equality by chance concerning both sub-samples is ensured.

The randomisation process of patients will be planned together with the staff of the participating rehabilitation centres, especially the medical directors, administration staff, system administrator and those responsible for therapy planning in the rehabilitation centres during the phase of preparation and implementation. For the planning of the randomisation process, the implementation of the data base and the training of administration staff to handle the data base, the rehabilitation centres will be visit two to three times.

\section{Sample size and power calculation}

Thinking of the short-term effectiveness of both interventions, only small differences between groups and small effect sizes at the end of rehabilitation ( $t 2)(\mathrm{d}=0,3$ or $f(V)=0,15)$ are expected to be found $[12,41,42]$. Because of the minor long-term effects of the present usual care, medium effect sizes $(\mathrm{d}=0,5$ or $\mathrm{f}(\mathrm{V})=0,25)$ are expected to be found at six and twelve months. This is in line with Friedrich and colleagues [43,44], who were able to show medium effect sizes regarding a reduction of disability when comparing a functional exercise therapy to an exercise therapy which contained additional motivational strategies (Disability Score: $\mathrm{d} \sim 0,68$ ). Sample power (Software: G-Power 3.0) concerning the primary outcome was approximated for an analysis of covariance at $\mathrm{t} 4$ with medium effect size of Cohen's $\mathrm{d}=0,5$, alpha error $5 \%$ and test power of $80 \%$. This approximisation results in a sample size of at least 128 patients without missing values. Anticipating a dropout rate of $40 \%$, it is necessary to include 214 patients ( $n=107$ for each group).

\section{Interventions \\ Control arm}

Both of the participating rehabilitation centres have a conceptually similar BMR for patients with orthopedic functional impairment and concurrent substantial psychological or social component of this impairment. The behavioural medical concept takes into account the biopsychosocial mechanisms relevant for the chronification of musculoskeletal disorders [9,45]. Empowerment and self-management are encouraged. On average, the BMR lasts for 27 days. Compared to the traditional multidisciplinary inpatient rehabilitation, the BMR has specifically defined inclusion criteria [11]: 1) an interdisciplinary admission, 2) a standardized psychosocial assessment, 3) a reconciled interdisciplinary case management, 4) case reviews on a regular basis, 5) supervision, 6) closed groups (a group remains together in all treatments during their whole stay at the rehabilitation centre), 7) the possibility of an individually tailored rehabilitation schedule and 8) therapist 
consistency for the most part (a therapist accompanies a group throughout a whole intervention). The multiprofessional core of the BMR relies on psychological interventions and standard exercise therapy (SET), in which ten to twelve patients take part together as a closed group. Other interventions are presentations with information about health and health behavior, rehabilitation/social counseling and if necessary occupational therapy. The total extent of therapy during the stay in the rehab centre is 65 hours on average, including approximately 26 hours exercise therapy. An experienced orthopedist bears the overall responsibility. A detailed description of the psychological group and exercise therapy group is available in the Additional files (see Additional file 1).

\section{Intervention arm}

Participants in the intervention arm will also receive a BMR, but with a specific behaviour-oriented exercise therapy (BET), which replaces SET. BET has been developed on the basis of previous work [46]. In general, BET aims at facilitating health-related knowledge and skills, which empower patients to maintain a healthy, physically active lifestyle beyond their BMR treatment. It's based on the assumption that a theory-based, systematic and integrated targeting of relevant psychosocial factors in the chronification of low back pain and also determinants of health behavior change, as well as factors of physical deconditioning and disuse improves the self-management and therefore reduces functional impairment of patients with clbp. This is reached by a structured combination of exercise, education and behavioural elements with a strong emphasis on patients' preferences and their previous experiences with exercise. Therefore different types of exercise are considered for patients to become familiar with their execution (e. g. methods of controlling intensities and training progression), as well as to encourage positive experiences with exercise. BET is delivered in closed groups with six to twelve patients during the approximately four weeks of the BMR. In total, exercise therapy comprised an extent of 26 hours on average. Both the fifteen sessions and the related modules of BET are guided by the same trained BET-therapist.

\section{BET sessions, 60 minutes duration}

The major part of BET consists of fifteen sessions with duration of 60 minutes each. Every single session comprises an introduction (approx. 5min), structured active play (approx. 5min), short education periods on selected topics (approx. 10min), exercises and relaxation (approx. 30min) as well as conclusion and sporadically a metaphoric story (approx. 10min). The entire thematic topic overview of the fifteen sessions can be seen in the in the Additional files (see Additional file 2).

\section{BET related modules, 20 to 60 minutes duration}

In the first week patients receive, two $60 \mathrm{~min}$. introductions in weight-lifting training, two $60 \mathrm{~min}$. introductions in an aerobic exercise (walking, nordic walking and ergometer cycling) and two $30 \mathrm{~min}$. introductions in aqua training (aqua aerobics, aqua jogging). Further three modules of planning out of every 60 minutes duration are distributed over three weeks. The purpose of these related modules is for patients to become familiar with different types of exercises in the first week supervised by a BET-therapist followed by a more self-directed planning and performing of exercises during the rehabilitation stay. Therefore during the four weeks of treatment the supervision by the therapist decreases, while the performing of selfdirected exercises increases. At the end of the second week, the BET-therapist will reflect together with the group reviewing, how well the individual realisation of planned exercises itself is managed. If barriers occur, they will be discussed and alternative possibilities will be developed. To be available for consult in case of any questions especially related to weightlifting training, additional three appointments will be scheduled (one each in the 2nd to 4 th week of therapy) under supervision of a BET therapist. Table 2 shows the volume of related modules with duration, frequency and distribution over the four weeks of the rehabilitation phase.

In summary, the following key features characterized BET:

- Patient-centred approach through active participation of patients in the entire treatment

Table 2 BET related modules

\begin{tabular}{lll}
\hline BET related modules & Duration & Frequency/ week of rehabilitation \\
\hline Weight-lifting training introduction & $60 \mathrm{~min}$ & 2x/ week 1 \\
\hline Weight-lifting training & $60 \mathrm{~min}$ & $3 \times$ distributes over week 2 to 4 \\
\hline Aerobic exercise introduction & $60 \mathrm{~min}$ & $2 \times /$ week 1 \\
\hline Aquatic training introduction & $30 \mathrm{~min}$ & $2 \times /$ week 1 \\
\hline Planning module & $60 \mathrm{~min}$ & $3 \times$ distributes over week 1 and 2 \\
\hline Individual physical activity/ exercise & -- & self-directed physical activity/ exercise distributes over week 2 to 4 \\
\hline
\end{tabular}


process and considering their individual preferences and previous experiences with exercise

- Goal-oriented combination of exercise, education, and behavioural elements within the BET

- Psychosocial factors that contribute to the chronification of clbp (e. g. pain-related cognitions, avoidance behavior, endurance behaviour) are systematically modified by the use of structured contents and methods which refer to exercise, education and behavioural elements

- Theory-based use of behavioural techniques to influence motivational and volitional determinants of health behavior change to promote long-term maintenance of self-directed physical activity

- Specific patient-oriented, plain media and materials with a very demanding character are used

- Trainer manual with a detailed description of the BET program for a standardized execution of the exercise, educational and behavioural elements, as well as a standardized use of media and materials to achieve multidimensional objectives of the BET is available

- Objectives, contents and methods of BET are matched in terms of a consistent approach and cooperation of all professions involved in the BMR

- Key messages and competencies trained by medical and psychological professions in the BMR will be reflected and reinforced within the BET.

More information can be found in the Additional files (see Additional file 3) and the detailed trainer manual is available in German and can be requested from the authors.

\section{Treatment adherence}

The therapists who perform BET in the intervention group will receive an intensive training on the objectives, contents, structure, components and methods of the program. During the implementation phase two workshops (each 2 days with 720 min duration) at intervals of eight weeks will take place together with all BET-therapists from both rehabilitation centres. The workshops are provided on the basis of a manualised intervention concept and are delivered by the same trainers. Moreover, pilot groups with the BET program will be performed within the implementation phase. The BET-therapists are skilled in the use of the program and try out the approach with the specific goal-orientated components as well as the media and materials.

The intensive training of BET-therapists and the implementation of the BET-groups will be supervised and evaluated. For data collection, quantitative methods are used (self-constructed questionnaires). The acceptance of the therapists (motivation, readiness to change, and program satisfaction) and difficulties during the implementation as well as acceptance of the patients (program satisfaction) will be recorded.

Both exercise programs (SET and BET) within the BMR have the same duration. The duration of both exercise programs as well as additional interventions within the BMR are documented for each study patient during the intervention phase.

A potential source of bias in the execution of RCT in the inpatient rehabilitation is the potential mixture between the study group and the control group through a communication about intervention contents between patients as well as therapists within each rehabilitation centre. The adaptations in the BET aimed at the integration of behavioural techniques and a modified use of teaching approaches. A reflected effective exchange of the adapted intervention contents requires extensive knowledge of the underlying theoretical models of the chronification of lbp. This is generally not expected from patients of a BMR. In addition, the patients of both study groups will be informed that both exercise programs within in the BMR are of high quality and will be delivered based on actual scientific evidence.

Trained BET-therapists will sign a written contract concerning discretion about objectives, contents, structure, methods, media and materials used within the BET. Regularly, announced visitations during the intervention phase will occur in order to assure adherence to the treatment protocol. Individual deviations from the treatment protocol will be recorded. In the case of vacation or sick leave, a trained BET-therapist for replacement is available.

\section{Outcome assessment}

Standardised questionnaires will be used to measure the primary and secondary outcomes. Our choice of outcome measures is based on two previous trials of our working group to evaluate effectiveness of different interventions in the management of clbp $[38,46]$. They are also in line with international recommendations [47-50]. All outcomes measures and their measuring time are shown in Table 3.

\section{Primary outcome measure}

We will use the level of functional limitations associated to clbp twelve months after the end of BMR measured by the Hannover Functional Ability Questionnaire (FFbH-R) as our primary outcome [51]. The FFbH-R consists of twelve items with a three-stage answering scale. Its summary score describes the low back pain associated functional ability in activities of daily living in adults on a scale of $0 \%$ (minimum functional ability) to $100 \%$ (maximum functional ability). The questionnaire is constructed for response to already light and moderate functional restrictions. The average item intercorrelation amounts to 0.50 . The test-retest-reliability with repeated measures after 
Table 3 Primary and secondary outcomes

\begin{tabular}{|c|c|c|c|c|c|c|}
\hline \multirow{2}{*}{$\begin{array}{l}\text { Domain/outcome } \\
\text { Primary Outcome }\end{array}$} & \multirow[t]{2}{*}{ Questionnaire/items } & \multicolumn{4}{|c|}{$\begin{array}{l}\text { Assessment } \\
\text { time point }\end{array}$} & \multirow[t]{2}{*}{ Reference } \\
\hline & & $\mathrm{t} 1$ & t2 & t3 & t4 & \\
\hline Physical functioning & Hannover Functional Ability Questionnaire (FfbH-R) & $x$ & $x$ & $\mathrm{x}$ & $x$ & [51] \\
\hline \multicolumn{7}{|l|}{ Secondary Outcomes } \\
\hline \multicolumn{7}{|l|}{ Pain } \\
\hline Pain intensity & Numeric Rating Scale (NRS) & $x$ & $x$ & $x$ & $x$ & [52] \\
\hline Pain status & Graded Chronic Pain Status (GCPS) & $x$ & $x$ & $x$ & $x$ & [53] \\
\hline Physical activity & Freiburg questionnaire on physical activity (FFkA) & $x$ & & $\mathrm{x}$ & $x$ & [54] \\
\hline \multicolumn{7}{|l|}{ Emotional functioning } \\
\hline Depression & Patient Health Questionnaire (PHQ-D) & $x$ & $x$ & $x$ & $x$ & [55] \\
\hline Anxiety & Generalized anxiety disorder 7-item scale (GAD-7) & $x$ & $x$ & $x$ & $x$ & [56] \\
\hline Health related quality of life & Short-Form-12 (SF-12) & $x$ & $x$ & $x$ & $x$ & [57] \\
\hline Stress & Perceived Stress Scale (PSS) & $x$ & $x$ & $x$ & $x$ & [58] \\
\hline \multicolumn{7}{|l|}{$\begin{array}{l}\text { Pain related cognitions, } \\
\text { emotions and behavior }\end{array}$} \\
\hline $\begin{array}{l}\text { Cognitive and behavioral pain } \\
\text { coping strategies }\end{array}$ & Pain Management Questionnaire (FESV) & $x$ & $x$ & $\mathrm{x}$ & $x$ & [59] \\
\hline $\begin{array}{l}\text { Fear-avoidance and endurance- } \\
\text { related responses to pain }\end{array}$ & Avoidance-Endurance Questionnaire (AEQ) & $x$ & $x$ & $x$ & $\mathrm{x}$ & {$[60]$} \\
\hline Pain related fear & Tampa Scale for Kinesiophobia (TSK) & $x$ & $x$ & $x$ & $x$ & {$[61,62]$} \\
\hline \multicolumn{7}{|l|}{$\begin{array}{l}\text { Motivational and volitional } \\
\text { determinants of physical activity }\end{array}$} \\
\hline & $\begin{array}{l}\text { risk perception ( } 3 \text { items), self-efficacy ( } 3 \text { items), outcome expectancies ( } 11 \text { items), } \\
\text { experiences with physical activity ( } 5 \text { items), self-concordance ( } 12 \text { items), intention } \\
\text { ( } 7 \text { items), action ( } 4 \text { items) and coping ( } 4 \text { items) planning, action control (6 items) }\end{array}$ & $x$ & $x$ & $x$ & $x$ & {$[63-67]$} \\
\hline $\begin{array}{l}\text { Stage of change algorithm for } \\
\text { physical activity }\end{array}$ & $\begin{array}{l}\text { two Items (Have you performed moderate physical for } 30 \text { minutes or longer on } \\
\text { a minimum of } 3 \text { days per week? and "Since when are you as regularly active as } \\
\text { you are now?"; possible answers: 1. Question: no, and I don't intend to do so; no, } \\
\text { but I am thinking about doing so; no, but I have the strong intention to do so; } \\
\text { yes, but it is difficult to me; yes, and it is easy for me; 2. Question: open) }\end{array}$ & $x$ & $x$ & $x$ & $x$ & {$[68,69]$} \\
\hline $\begin{array}{l}\text { Attitudes towards performing } \\
\text { physical activity }\end{array}$ & $\begin{array}{l}\text { modified Version of Short-Questionnaire to measure cognitive and affective } \\
\text { attitudes toward sports activities }\end{array}$ & $x$ & $x$ & $x$ & $\mathrm{x}$ & [70] \\
\hline $\begin{array}{l}\text { Treatment satisfactions with } \\
\text { exercise therapy }\end{array}$ & $\begin{array}{l}18 \text { items (e.g. "I would recommend the exercise therapy other patients." } \\
\text { measured by using a six-point ordinal scale with } 1=\text { "absolutely agree", } \\
6=\text { "absolutely disagree"; e.g. "The time extent of the exercise therapy, I perceived } \\
\text { as..." measured by using a five-point ordinal scale with } 1=\text { "far too high", } 5=\text { "far } \\
\text { too low"; e.g. "Overall, how do you rate the exercise therapy?" measured by using } \\
\text { a six-point ordinal scale with 1= "very satisfied", 6=not "satisfied at all") }\end{array}$ & & $x$ & & & self-designed \\
\hline $\begin{array}{l}\text { Treatment satisfactions with the } \\
\text { rehabilitation process }\end{array}$ & $\begin{array}{l}\text { five items (e.g. "Overall, how do you rate the rehabilitation process?") measured } \\
\text { by using a ten-point ordinal scale ( } 1=\text { "very poor", } 10={ }_{\text {"excellent") }}\end{array}$ & & $x$ & & & self-designed \\
\hline \multicolumn{7}{|l|}{ Others } \\
\hline Demographic characteristics & $\begin{array}{l}\text { sex, age, height, nationality, marital status, education, monthly income, weight, } \\
\text { self-reported work status }\end{array}$ & $x$ & & & & [71] \\
\hline \multirow[t]{3}{*}{ Social medical characteristics } & $\begin{array}{l}\text { a) self-reported low back pain related sick leave, use of health care services, } \\
\text { medication use for the last six month and self-reported plan to apply for } \\
\text { retirement, severe disabilities }\end{array}$ & $x$ & & & & [71] \\
\hline & $\begin{array}{l}\text { b) diagnosis, work status and physical capability taken from the hospital discharge } \\
\text { report }\end{array}$ & & $x$ & & & \\
\hline & a) aftercare recommendations taken from the hospital discharge report & & $x$ & & & self-designed \\
\hline Aftercare & $\begin{array}{l}\text { b) self-designed; five items (e.g. "Have you participated in the last twelve months } \\
\text { in a medically prescribed exercise therapy to rehabilitation aftercare?" with the } \\
\text { option to answer yes/no) }\end{array}$ & & & & $x$ & \\
\hline Job satisfaction & scale on job satisfaction (IRES-3) & & & $x$ & $x$ & [72] \\
\hline
\end{tabular}


approximately one week is above 0.75 , Cronbach alpha figures 0.90 [51]. The one factorial structure of the instrument was confirmed in a principal component analysis. The FFbH-R is comparable to the internationally used Roland Morris Questionnaire for measuring clbp related disability with a correlation of 0.75 [73].

\section{Secondary outcome measures}

Secondary outcome measures refer to demographics and related characteristics, pain, physical activity, healthrelated quality of life, depression, anxiety, stress, painrelated cognitions, emotions and behavior, motivational and volitional determinants of physical activity, attitudes towards performing physical activity, satisfaction with exercise therapy and satisfaction with the rehabilitation process (see Table 3).

\section{Data analysis}

Data analysis is carried out following evaluation standards of experimental study designs with control groups, using descriptive methods as well as inferential statistics. An intention-to-treat analysis will be performed. In the case of dropouts and withdrawals, multiple imputation techniques will be used. Baseline differences in demographic as well as the primary and secondary outcomes data will be analysed using two-sample t-tests for parametric and Wilcoxon test for non-parametric distribution as well as Chi-Square test for nominal data. The testing of the hypotheses is conducted via comparison of changes in the intervention and the control group regarding the primary and secondary outcomes. For this purpose, a saturated $4 \times 2$-factorial linear mixed effects model in the process of all four assessment time points and two groups as fixed effects and intercept and slope as random effects is used whilst controlling for statistically significant differences at baseline [74]. Changes in outcomes over time are represented by their linear slopes. Because of the disparity between the duration of the inpatient rehabilitation phase (27 days on average) and the follow-up phase (12 months), separate slopes for these phases are estimated by a spline model. Primarily overall group differences are identified by a model comparing likehood ratio test of a hypothesized model incorporating interaction effects of group by time and a nested null model leaving out these interactions. Additionally the slopes of both groups can be compared in two phases whilst controlling for statistically significant differences at baseline. Furthermore, a post hoc analysis using similar mixed effects linear regression techniques is conducted to model effects of secondary outcomes onto the primary outcome to explore possible interactions.

\section{Blinding}

Blinding of the therapists is not possible because they will be trained to perform the BET or will be chosen deliver the existing exercise therapy within the BMR during the study period.

Patients will be masked regarding the study group. They will be informed through the staff and in the "informed consent" that the effectiveness of two exercise therapy programs will be compared and that both meet current scientific standards and are appropriate to improve health status. During the study period, patients will be not informed as to whether they participate in the control group or intervention group.

A person who will not be involved in the study process performs the statistical data analysis. Furthermore, the evaluation will be blinded to the treatment group.

\section{Ethical aspects}

Ethical and legal problems are not anticipated because of experiences with comparable studies. This investigation is conducted according to the recommendations of the World Medical Association (Declaration of Helsinki: [75]). Information for the participants and their agreement on study participation is written down as "informed consent". It is pointed out to the participants that the study participation is voluntary and that they may refuse to participate or to discontinue participation at any time without disadvantages or loss of benefits. According to the national data protection laws all personal data is treated as confidential and is used only for scientific purposes. Ethical approval has been granted by the independent Research Ethics Committee of the Medical Faculty of Friedrich-AlexanderUniversity of Erlangen-Nürnberg (Re.-No. 4510). High ethical demands are also imposed by the study sponsor, the German Pension Insurance Association (Deutsche Rentenversicherung Bund). There is a special focus on data privacy.

\section{Discussion}

In the management of disabling clbp intensive multidisciplinary rehabilitation is recommended. But, the reported effects especially in the long-term are low $[8,10]$. A traditional approach of exercise therapy which is often deduced from physiological models is a cornerstone within multidisciplinary rehabilitation. With BMR, an intensified and theory-based rehabilitation program with proven shortterm effectiveness is available in Germany [12]. Exercisetherapy is a central component within BMR, but in practice a biomedical approach mainly to improve physical fitness has established which might work as an obstacle to enhance the long-term effects of a BMR. However, the growing empirical evidence for the importance of psychosocial factors in the development of clbp [19] and the weak relationship between changes in physical function measures and changes in various health outcomes in clbp after exercise therapy [76] requires a more behavioural approach of exercise therapy to facilitate self-management and 
adherence to regular physical activity and therefore reducing pain and disability in the long-term [22]. Only two exercise therapy programs with a theory-based and behavioural approach are available for patients with clbp in Germany, both with a focus to enhance exercise adherence $[31,32]$. The majority of exercise therapy programs in the routine of traditional multidisciplinary inpatient rehabilitation in Germany are not explicitly theory-based, so it is unclear which factors of the chronification process and causal mechanism of clbp are influenced. On the other hand, causal mechanisms remain inadequately clarified, even in theoretical models for clbp $[20,77,78]$. The evaluation of theory-based exercise therapy programs can contribute to the further development of theoretical assumptions of the chronification process of clbp.

Therefore, there is a need for the development, implementation and evaluation of a theory-based exercise therapy with a behavioural approach (BET) in the context of a BMR. To our knowledge, this is the first RCT to examine the effects of BET for the overall treatment success of BMR in the routine healthcare management of patients with disabling clbp.

Methodological challenges arise from conducting a RCT, which claims a lot of resources, within routine health care in two rehabilitation centres as well as from ensuring high treatment integrity. The latter refers particularly to a potential mixture between the study group and the control group through a communication about intervention contents between patients and therapists within each rehabilitation centre, as well as individual deviations from the treatment protocol. To overcome those potential sources of bias BET is delivered by the same BET-therapist, who will be intensively trained in the use of the program and will receive a detailed trainer manual. BET-therapists will sign a written contract concerning discretion about BET. Regularly, announced visitations during the intervention phase will occur in order to assure adherence to the treatment protocol. Individual deviations from the treatment protocol will be recorded.

Altogether, findings of this study might contribute to a better understanding of the mechanism of action of BMR and the special effects of BET and might be used to improve the quality of these interventions in routine care and therefore reduce the burden to patients with clbp.

\section{Additional file}

Additional file 1: Description of two main interventions within BMR - psychological group therapy and standard exercise therapy (SET) in both participating rehabilitation centres (control group).

Additional file 2: Intervention group.

Additional file 3: BET_Therapy plan.

\section{Abbreviations}

BMR: Behavioural medical rehabilitation; BET: Behavioural exercise therapy; clbp: Chronic non-specific low back pain; min: Minutes; RCT: Randomised controlled trial; SET: Standard exercise therapy.

\section{Competing interests}

The authors declare to have no competing interests.

\section{Authors' contributions}

$J \mathrm{H}$ drafted the manuscript and contributed substantially to the development of the research questions, the study design and the study protocol in general. Prior to all of that, JH was also responsible for conducting an indepth literature search to prepare the BET and the study as a whole. $\mathrm{JH}$ is further responsible for the study organisation and oversees patient enrollment and data management. $\mathrm{JH}$ is also the coach at the train-the -trainer seminar, who teaches the main part. SP contributed to the development of the assessment tools for the research questions and was a major contributor to the creation of the BET and also teaches several parts at the above mentioned train-the-trainer seminar. SP wrote the respective chapters of this manuscript and contributed substantially to the other chapters. $\mathrm{CH}$ contributed substantially to the determination of primary and secondary outcomes and the according assessment tools. $\mathrm{CH}$ further oversaw the creation of the study design and is the main contributor regarding randomization, data management and data analysis. WG contributed substantially to the development of the research questions, the study design and the development of contents, methods and materials of BET. KP conceived the study and was responsible for identifying the research question beforehand. KP drafted the study design and was accountable for determining primary and secondary outcomes. KP contrived the basis for development of the experimental treatment. Every author read and approved the final version of this paper.

\section{Acknowledgements}

The authors thank the German Pension Insurance Association (Deutsche Rentenversicherung Bund), which sponsors and supports this study within the Research funding for care-oriented research "Chronic disease and Patientorientation" in module two of the second funding phase [79]. We also thank Dr. med. Désirée Herbold (chief physician), (rehabilitation centre "Paracelsus-Klinik an der Gande"), Dr. med. Martin Holme (medical director), Dr. med. Gabriele Sohr (assistent medical director) (rehabilitation centre "Klinik Weser Bad Pyrmont") and all the practitioners in both institutions. We also thank all the therapists (For the "Paracelsus-Klinik an der Gande": Frank Härdter, Arndt Buttgereit and Dana Voigt; For the "Klinik Weser Bad Pyrmont": Gabriele Voß, Maria Wilmes, Ute Müller, Maike von der Heide and Gabriele Engelsmann) and the entire team of physical therapists in both institutions for their outstanding engagement. We further thank all secretaries (For the "Paracelsus-Klinik an der Gande": Janina Laake and Kirsten Fischer; For the "Klinik Weser Bad Pyrmont": Martina Brinkmann and Ute Schuster), therapy planners (For the "Paracelsus-Klinik an der Gande": Sascha Meyer; For the "Klinik Weser Bad Pyrmont": Corinna Lange), and others, who are in any way engaged in this study. We further thank Mandy Philipps for the language revision of this manuscript.

Received: 30 January 2013 Accepted: 28 February 2013 Published: 11 March 2013

\section{References}

1. Andersson GBJ: Epidemiological features of chronic low-back pain. Lancet 1999, 354:581-585

2. Maniadakis N, Gray A: The economic burden of back pain in the UK. Pain 2000, 84:95-103.

3. Louw QA, Morris LD, Grimmer-Somers K: The prevalence of low back pain in Africa: a systematic review. BMC Musculoskelet Disord 2007, 8:105.

4. Balagué F, Mannion AF, Pellisé F, Cedraschi C: Non-specific low back pain. Lancet 2012, 379:482-491.

5. Schmidt CO, Raspe H, Pfingsten M, Hasenbring M, Basler HD, Eich W, Kohlmann T: Back Pain in the German Adult Population. Prevalence, Severity, and Sociodemographic Correlates in a Multiregional Survey. Spine 2007, 32(18):2005-2011.

6. Wenig CM, Schmidt CO, Kohlmann T, Schweikert B: Costs of back pain in Germany. Eur J Pain 2009, 13(3):280-286. 
7. Airaksinen O, Brox Jl, Cedraschi C, Hildebrandt J, Klaber-Moffett J, Kovacs F, Mannion AF, Reis S, Staal JB, Ursin H, Zanoli G: Chapter 4 European guidelines for the management of chronic nonspecific low back pain. Eur Spine J 2006, 15(Suppl. 2):S192-\$300.

8. Deutsche Rentenversicherung (German Pension Insurance Association): Rehabilitation 2007. Statistik der Deutschen Rentenversicherung (Statistics of the German Pension Insurance Association). Berlin: Deutsche Rentenversicherung Bund; 2008.

9. Bundesärztekammer (BÄK), Kassenärztliche Bundesvereinigung (KBV), Arbeitsgemeinschaft der Wissenschaftlichen Medizinischen Fachgesellschaften (AWMF): Nationale VersorgungsLeitlinie Kreuzschmerz Langfassung (Long version of the national guideline for the management of low back pain). 2011. Version 1.2. 2011, accessed: 10/13/2011. [http://www. versorgungsleitlinien.de/themen/kreuzschmerz]

10. Van Middelkoop M, Rubinstein SM, Kuijpers T, Verhagen AP, Ostelo R, Koes BW, van Tulder MW: A systematic review on the effectiveness of physical and rehabilitation interventions for chronic non-specific low back pain. Eur Spine J 2011, 20:19-39.

11. Deutsche Rentenversicherung Bund (German Pension Insurance Association): Positionspapier zum Konzept der verhaltensmedizinisch orthopädischen Rehabilitation (VMO) (Position paper to the concept of the behavioural medical rehabilitation (BMR)). 2012. unpublished paper.

12. Mangels M, Schwarz S, Worringen U, Holme M, Rief W: Evaluation of a behavioral-medical inpatient rehabilitation treatment including booster sessions: a randomized controlled study. Clin J Pain 2009, 25:356-364

13. Bethge $M$, Müller-Fahrnow W: Wirksamkeit einer intensivierten stationären Rehabilitation bei muskuloskelettalen Erkrankungen: systematischer Review und Meta-Analyse (Efficacy of Intensified Inpatient Rehabilitation in Musculoskeletal Disorders: Systematic Review and Meta-Analysis). Rehabilitation 2008, 47(4):200-209.

14. Scascighini L, Toma V, Dober-Spielmann S, Sprott H: Multidisciplinary treatment for chronic pain: a systematic review of interventions and outcomes. Rheumatology 2008, 47:670-678.

15. Brüggemann S, Sewöster D: Bewegungstherapeutische Versorgung in der medizinischen Rehabilitation der Rentenversicherung (Exercise therapy in the medical rehabilitation of German Pension Insurance Association). Bewegungstherapie und Gesundheitssport 2010, 26:266-269.

16. Arbeitsgruppe Bewegungstherapie ("Exercise Therapy" Commission): Ziele und Aufgaben der Arbeitsgruppe "Bewegungstherapie" in der Deutschen Gesellschaft für Rehabilitationswissenschaften (DGRW) (Objectives and Tasks of the "Exercise Therapy" Commission of the German Society of Rehabilitation Science, DGRW). Rehabilitation 2009, 48:252-255.

17. Hayden JA, van Tulder MW, Malmivaara A, Koes BW: Exercise therapy for treatment of non-specific low back pain. Cochrane Database Syst Rev 2005. CD000335. doi:10.1002/14651858.CD000335.pub2.

18. Van Middelkoop M, Rubinstein SM, Verhagen AP, Ostelo RW, Koes BW, van Tulder MW: Exercise therapy for chronic nonspecific low-back pain. Best Pract Res Clin Rheumatol 2010, 24(2):193-204.

19. Leeuw M, Goossens ME, Linton SJ, Crombez G, Boersma K, Vlaeyen JW: The fear-avoidance model of musculoskeletal pain: current state of scientific evidence. J Behav Med 2007, 30(1):77-94.

20. Pincus T, Smeets RJEM, Simmonds MJ, Sullivan MJL: The fear avoidance model disentangled: improving the clinical utility of the fear avoidance model. Clin J Pain 2010, 26(9):739-746.

21. Hasenbring MI, Verbunt JA: Fear-avoidance and Endurance-related Responses to Pain: New Models of Behavior and Their Consequences for Clinical Practice. Clin J Pain 2010, 26(9):747-753.

22. Nicholas MK, George SZ: Psychologically informed interventions for low back pain: an update for physical therapists. Phys Ther 2011, 91:765-776

23. Main CJ, George SZ: Psychologically informed practice for management of low back pain: future directions in practice and research. Phys Ther 2011, 91:820-824.

24. Jordan JL, Holden MA, Mason EEJ, Foster NE: Interventions to improve adherence to exercise for chronic musculoskeletal pain in adults. Cochrane Database Syst Rev 2010. CD005956. doi:10.1002/14651858.CD005956.pub2.

25. Dean E, Al-Obaidi S, de Andrade AD, Gosselink R, Umerah G, Al-Abdelwahab S, Anthony J, Bhise AR, Bruno S, Butcher S, Fagevik-Olsén M, et al: The First Physical Therapy Summit on Global Health: Implications and Recommendations for the $21^{\text {st }}$ century. Physiother Theory Pract 2011, 27(8):531-547.
26. Linton SJ, Shaw WS: Impact of psychological factors in the experience of pain. Phys Ther 2011, 91:700-711.

27. Conn VS, Hafdahl AR, Brown SA, Brown LM: Meta-analysis of patient education interventions to increase physical activity among chronically ill adults. Patient Educ Couns 2008, 70(2):157-172.

28. Ruppar TM, Conn VS: Interventions to promote physical activity in chronically ill adults. Am J Nurs 2010, 110(7):30-37.

29. Hayden JA, van Tulder MW, Tomlinson G: Systematic review: strategies for using exercise therapy to improve outcomes in chronic low back pain. Ann Intern Med 2005, 142(9):776-785.

30. Hofmann J, Böhle E, Bork H, Brüggemann S, Greitemann B, Hildebrandt J, Kladny B, Pfeifer K: Best-Practice-Empfehlungen zu Zielsetzungen, Inhalten und Methoden ambulanter und stationärer Rehabilitationsmaßnahmen von Patienten mit chronifizierenden oder chronischen Rückenschmerzen (Best-Practice-Recommendations for Objectives, Contents and Methods in the outpatient and inpatient Rehabilitation of Chronic Low Back Pain). Phys Med Rehab Kuror 2010, 20:32-39.

31. Göhner W, Seelig H, Fuchs R: Intervention Effects on Cognitive Antecedents of Physical Exercise: A 1-Year Follow-Up Study. Applied Psychology: Health and Well-being 2009, 1(2):233-256.

32. Lippke S, Ziegelmann JP, Schwarzer R: Behavioral Intentions and Action Plans Promote Physical Exercise: A Longitudinal Study With Orthopedic Rehabilitation Patients. J Sport Exerc Psychol 2004, 26:470-483.

33. Biddle S, Fuchs R: Exercise psychology: A view from Europe. Psychol Sport Exerc 2009, 10:410-419.

34. Van der Hulst M, Vollenbroek-Hutten MM, Schreurs KM, Rietman JS, Hermens HJ: Relationships between coping strategies and lumbar muscle activity in subjects with chronic low back pain. Eur J Pain 2010, 14(6):640-647.

35. Leonhardt C, Keller S, Becker A, Luckmann J, Baum E, Donner-Banzhoff N, Pfingsten M, Hildebrandt J, Chenot JF, Kochen MM, Basler HD: Depressivität, Bewegungsangst-Kognitionen und körperliche Aktivität bei Patienten mit Rückenschmerz (Depression, fear-avoidance beliefs, and physical activity in patients with low back pain). Z Sportpsychol 2007, 14(1):29-43

36. Crowe M, Whitehead L, Jo Gagan M, Baxter D, Panckhurst A: Selfmanagement and chronic low back pain: a qualitative study. J Adv Nurs 2010, 66(7):1478-1486.

37. Scholich SL, Hallner D, Wittenberg RH, Hasenbring MI, Rusu AC: The relationship between pain, disability, quality of life and cognitivebehavioural factors in chronic back pain. Disabil Rehabil 2012, 34(23):1993-2000

38. Hentschke C, Hofmann J, Pfeifer K: A bio-psycho-social exercise program (RÜCKGEWINN) for chronic low back pain in rehabilitation aftercare Study protocol for a randomised controlled trial. BMC Musculoskelet Disord 2010, 11:266.

39. Stout RL, Wirtz PW, Carbonari JP, del Boca FK: Ensuring balanced distribution of prognostic factors in treatment outcome research. J Stud Alcohol 1994, 55(Suppl. 12):70-75.

40. Wei $L$, Lachin JM: Properties of the urn randomization in clinical trials. Control Clin Trials 1988, 9(4):345-364.

41. Greitemann B, Dibbelt S, Büschel C: Integriertes OrthopädischPsychosomatisches Konzept zur medizinischen Rehabilitation von Patienten mit chronischen Schmerzen des Bewegungsapparats Langfristige Effekte und Nachhaltigkeit eines multimodalen Programms zur Aktivierung und beruflichen Umorientierung (Multidisciplinary Orthopedic Rehabilitation Program in Patients with Chronic Back Pain and Need for Changing Job Situation - Long-Term Effects of a Multimodal, Multidisciplinary Program with Activation and Job Development). Z Orthop 2006, 144:255-266.

42. Hüppe A, Raspe H: Zur Wirksamkeit stationärer medizinischer Rehabilitation in Deutschland bei chronischen Rückenschmerzen: Aktualisierung und methodenkritische Diskussion einer Literaturübersicht (Efficacy of Inpatient Rehabilitation for Chronic Back Pain in Germany: Update of a Systematic Review). Rehabilitation 2005, 44:24-33.

43. Friedrich M, Gittler $G$, Halberstadt $Y$, Cermak T, Heiller I: Combined exercise and motivation program: effect on the compliance and level of disability of patients with chronic low back pain: a randomized controlled trial. Arch Phys Med Rehabil 1998, 79(5):475-487. 
44. Friedrich M, Gittler $G$, Arendasy M, Friedrich KM: Long-term effect of a combined exercise and motivational program on the level of disability of patients with chronic low back pain. Spine 2005, 30(9):995-1000

45. Waddell G: The biopsychosocial model. In The Back pain Revolution. 2nd edition. Edited by Waddell G. Edinburgh: Churchill Livingston; 2004:265-282.

46. Pfeifer K, Hofmann J, Buchmann J, Meng K, Vogel H, Faller H, Bork H: Langfristige Wirksamkeit einer integrativen Patientenschulung zur Optimierung der stationären Rehabilitation bei chronischem Rückenschmerz (PASTOR) - eine multizentrische, prospektive Kontrollgruppenstudie (Long-term effectiveness of an integrative patient education to the optimisation of inpatient rehabilitation in chronic lowback pain (PASTOR) - a prospective study with a control group). In Tagungsband des 21. Edited by Deutsche Rentenversicherung Bund. Hamburg: Rehabilitationswissenschaftlichen Kolloquiums: 5.-7. März 2012; 2012:370-373

47. Chapman JR, Norvell DC, Hermsmeyer JT, Bransford RJ, DeVine J, McGirt MJ, Lee MJ: Evaluating Common Outcomes for Measuring Treatment Success for Chronic Low Back Pain. Spine 2011, 36(21S):S54-S68.

48. Dworkin RH, Turk DC, Wyrwich KW, Beaton D, Cleeland CS, Farrar JT, Haythornthwaite JA, Jensen MP, Kerns RD, Ader DN, et al: Interpreting the clinical importance of treatment outcomes in chronic pain clinical trials: IMMPACT recommendations. J Pain 2008, 9(2):105-121.

49. Dworkin RH, Turk DC, Peirce-Sandner S, Baron R, Bellamy N, Burke LB, Chappell A, Chartier K, Cleeland CS, Costello A, et al: Research design considerations for confirmatory chronic pain clinical trials: IMMPACT recommendations. Pain 2010, 149(2):177-193.

50. Pincus T, Santos R, Breen A, Burton AK, Underwood M: A Review and Proposal for the Core Set of Factors for Prospective Cohorts in Low Back Pain: A Consensus Statement. Arthritis Rheum 2008, 59(1):14-24.

51. Kohlmann T, Raspe H: Der Funktionsfragebogen Hannover zur alltagsnahen Diagnostik der Funktionsbeeinträchtigung durch Rückenschmerzen (FFBH-R) (The Hannover Functional Ability Questionnaire for measuring back pain-related functional limitations (FFbH-R)). Rehabilitation 1996, 35:1-8

52. Farrar JT, Young JP, LaMoreaux L, Werth JL, Poole RM: Clinical importance of changes in chronic pain intensity measured on an 11-point numerical pain rating scale. Pain 2001, 94:149-158.

53. Von Korff M, Ormel J, Keele FJ, Dworkin SF: Grading the severity of chronic pain. Pain 1992, 50:133-149.

54. Frey I, Berg A: Physical activity counseling: Assessment of physical activity by questionnaire. Eur J Sport Sci 2002, 2(4):1-6.

55. Löwe B, Spitzer RL, Zipfel S, Herzog W: Gesundheitsfragebogen für Patienten (PHQ-D). Manual und Testunterlagen (Health questionnaire for patients (PHQD). Manual and test materials). 2nd edition. Karlsruhe: Pfizer; 2002.

56. Löwe B, Decker O, Müller S, Brähler E, Schellberg D, Herzog W, Herzberg PY: Validation and standardization of the Generalized Anxiety Disorder Screener (GAD-7) in the general population. Medical care 2008, 46(3):266-274.

57. Bullinger M, Kirchberger I: Der SF-36 Fragebogen zum Gesundheitszustan Handanweisung (The SF-36 questionnaire for measuring the health status manual). Göttingen: Hogrefe; 1998.

58. Cohen S, Williamson G: Perceived stress in a probability sample of the U. S. In The social psychology of health: Claremont Symposium on Applied Social Psychology. Edited by Spacapam S, Oskamp S. Newbury Park, CA: Sage; 1988:31-67

59. Geissner E: FESV-Fragebogen zur Erfassung der Schmerzverarbeitung (FESV-Questionnaire for the detection of pain coping strategies). Göttingen: Hogrefe; 2001

60. Hasenbring MI, Hallner D, Rusu AC: Fear-avoidance- and endurancerelated responses to pain: Development and validation of the Avoidance-Endurance Questionnaire (AEQ). Eur J Pain 2009, 13(6):620-628.

61. Kori SH, Miller RP, Todd DD: Kinesiophobia: a new view of chronic pain behavior. Pain Management 1990, 3:35-42.

62. Vlaeyen JW, Kole-Snijders AM, Boeren RG, van Eek H: Fear of movement/ (re)injury in chronic low back pain and its relation to behavioral performance. Pain 1995, 62:363-372.

63. Sniehotta FF, Scholz U, Schwarzer R: Bridging the intention-behaviour gap: Planning, self-efficacy, and action control in the adoption and maintenance of physical exercise. Psychol Health 2005, 20(2):143-160.
64. Sniehotta FF, Schwarzer R, Scholz U, Schüz B: Action planning and coping planning for long-term lifestyle change: Theory and assessment. Eur J Soc Psychol 2005, 35:565-576.

65. Fleig L, Lippke S, Pomp S, Schwarzer R: Exercise maintenance after rehabilitation: How experience can make a difference. Psychol Sport Exerc 2011, 12:293-299.

66. Seelig H, Fuchs R: Messung der sport- und bewegungsbezogenen Selbstkonkordanz (Measuring sport- and movement-related self concordance). Z Sportpsychol 2006, 13(4):121-139.

67. Scholz U, Sniehotta FF: Langzeiteffekte einer Planungs- und Handlungskontrollintervention auf die körperliche Aktivität von Herzpatienten nach der Rehabilitation (Long-term effects of a planning and action control intervention on physical activity of cardiac patients after rehabilitation). Zeitschrift für Gesundheitspsychologie 2006, 14(2):73-81.

68. Fleig L, Lippke S, Wiedemann AU, Ziegelmann JP, Reuter T, Gravert C: Förderung von körperlicher Aktivität im betrieblichen Kontext. Ein randomisiertes Kontrollgruppen-Design zur Untersuchung von stadienspezifischen Interventionseffekten (Promoting physical activity in a workplace setting: A randomized control group study of stagematched interventions). Zeitschrift für Gesundheitspsychologie 2010, 18(2):69-78

69. Lippke S, Ziegelmann JP, Schwarzer R, Velicer WF: Validity of Stage Assessment in the Adoption and Maintenance of Physical Activity and Fruit and Vegetable Consumption. Health Psychol 2009, 28(2):183-193.

70. Brand R: Die affektive Einstellungskomponente und ihr Beitrag zur Erklärung von Sportpartizipation (The role of the affective attitudinal component in explaining physical exercise participation). Z Sportpsychol 2006, 13(4):147-155.

71. Deck R, Röckelein E: Zur Erhebung soziodemographischer und sozialmedizinischer Indikatoren in den rehabilitationswissenschaftlichen Forschungsverbünden. Förderschwerpunkt "Rehabilitationswissenschaften". Empfehlungen der Arbeitsgruppen "Generische Methoden", "Routinedaten" und "Reha-Ökonomie" (For the assessment of sociodemographic and social-medical indicatiors in the research networks of rehabilitation science. Main funding area "rehabilitation science". Recommendations of the work groups "generic methods", "routine data" and "rehabilitation economics"). DRV-Schriften 1999, 16:84-102.

72. Bührlen B, Gerdes N, Jäckel WH: Entwicklung und psychometrische Testung eines Patientenfragebogens für die medizinische Rehabilitation (IRES-3) (Devolpment and psychometric properties of a questionnaires for patients for the medical rehabilitation). Rehabilitation 2005, 44:63-74

73. Roese I, Kohlmann T, Raspe H: Zur Messung der Funktionskapazität bei Rückenschmerzpatienten in der Rehabilitation: ein Vergleich standardisierter Fragebogen (Measurement of Functional Capacity in Rehabilitation Patients with Back Pain: Comparitive Analysis of Standardized Questionnaires). Rehabilitation 1996, 35:103-108.

74. Senn S, Stevens L, Chaturvedi N: Tutorial in Biostatistics. Repeated measures in clinical trials: simple strategies for analysis using summary measures. Stat Med 2000, 19:861-877.

75. World Medical Association (WMA): WMA Declaration of Helsinki - Ethical Principles for Medical Research Involving Human Subjects. http://www.wma. net/en/30publications/10policies/b3/17c.pdf.

76. Steiger F, Wirth B, De Bruin ED, Mannion AF: Is a positive clinical outcome after exercise therapy for chronic non-specific low back pain contingent upon a corresponding improvement in the targeted aspect(s) of performance? A systematic review. Eur Spine J 2012, 21(4):575-598.

77. Verbunt JA, Smeets RJ, Wittink HM: Cause or effect? Deconditioning and chronic low back pain. Pain 2010, 149(3):428-430.

78. Lundberg M, Frennered K, Hägg O, Styf J: The impact of fear-avoidance model variables on disability in patients with specific or nonspecific chronic low back pain. Spine 2011, 36(19):1547-1553.

79. Research funding for care-oriented research: Chronic diseases and Patientorientation. http://www.forschung-patientenorientierung.de/index. php/home.html.

doi:10.1186/1471-2474-14-89

Cite this article as: Hofmann et al: Effects of behavioural exercise therapy on the effectiveness of a multidisciplinary rehabilitation for chronic non-specific low back pain: Study protocol for a randomised controlled trial. BMC Musculoskeletal Disorders 2013 14:89. 